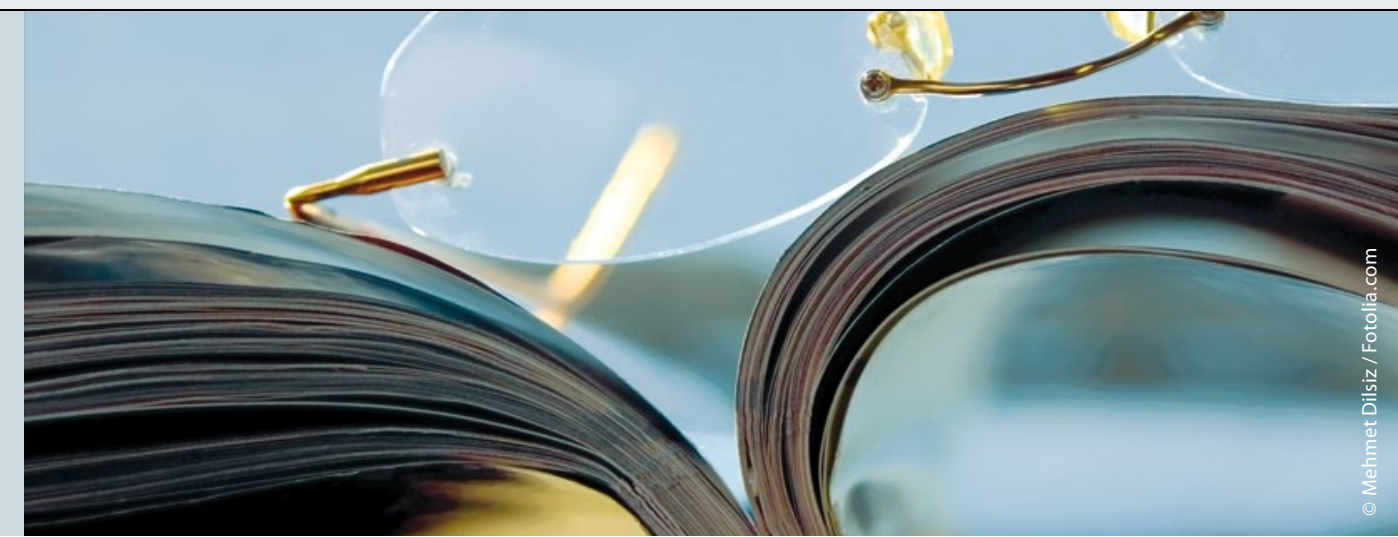

\title{
Folsäure zur Primärprävention des Schlaganfalls möglicherweise sinnvoll
}

\author{
Eine chinesische Studie konnte einen Nutzen der Folsäuregabe zusätzlich zu \\ Enalapril belegen. Ob dies auch für Kaukasier gilt, ist nicht geklärt.
}

mmer wieder sind Vitamingaben zur Primär-oder Sekundärprävention des Schlaganfalls untersucht worden. Letztlich hat sich nie ein überzeugendes Ergebnis gezeigt. Hintergrund sind Studien, die beim Schlaganfall einen Vitaminmangel oder eine Hyperhomocysteinämie gezeigt haben, die durch Vitamin B12 oder Folsäure ausgeglichen werden können.

In der hier vorliegenden chinesischen Studie wurden insgesamt 20.702 Patienten untersucht. Sie wurden zur Hypertoniebehandlung eingeschlossen und hatten bislang keinen Schlaganfall. Sie erhielten randomisiert entweder Enalapril $10 \mathrm{mg}$ allein oder mit 0,8 mg Folsäu- re zusätzlich. Insgesamt wurde das Kollektiv über viereinhalb Jahre beobachtet. In diesem Zeitraum erlitten 2,7\% der Patienten mit Folsäure und Enalapril und 3,4\% der Patienten, die nur Enalapril erhielten, einen Schlaganfall. Der Unterschied war statistisch signifikant. Auch andere Endpunkte (insbesondere die Kombination aus kardiovaskulärem Tod, Myokardinfarkt und Schlaganfall) unterschieden sich signifikant.

\section{Kommentar}

Dies ist die erste Studie, die randomisiert und doppelblind zeigen konnte, dass eine Gabe von Folsäure in der Primärprävention des Schlaganfalls möglicherweise sinnvoll ist. Bei der Interpretation müssen jedoch zwei Aspekte bedacht werden. Zum einen wurde eine chinesische Population untersucht. Hier könnten unter Umständen andere Stoffwechselprozesse und eine andere Metabolisierung vorliegen, sodass eine Übertragbarkeit auf Kaukasier nicht gewährleistet ist. Zum anderen galt die Risikoreduktion durch Folsäure nicht für Blutungen, sodass hierzu keine Aussage getroffen werden kann. Interessanterweise ist ja der Anteil der Blutungen bei Schlaganfällen in der asiatischen Bevölkerung deutlich höher als in der europäischen. Es bleibt also abzuwarten, ob ähnliche Studien auch eine Wirksamkeit von Folsäure (oder anderen Vitaminen) in der Primärprävention zeigen. Prof. Dr. med. Dr. phil. Stefan Evers

Huo Y, et al. CSPPT Investigators. Efficacy of folic acid therapy in primary prevention of stroke among adults with hypertension in China: the CSPPT randomized clinical trial. JAMA 2015; 313: $1325-35$

\section{Stenting plus Plättchenhemmung bei symptomatischer intrakranieller Stenose}

\author{
Bei intrakraniellen Stenosen bietet der Stent plus doppelte Plättchen- \\ hemmung keinen Vorteil gegenüber der rein medikamentösen Therapie - \\ die Kombination ist sogar eher unterlegen.
}

ntrakranielle Stenosen sind ein häufiger Grund für Hirninfarkte. Bei symptomatischen intrakraniellen Stenosen ( $\leq 70 \%)$ wurden trotz medikamentöser Therapie Reinfarktraten von bis zu $23 \%$ im ersten Jahr beschrieben.
In dieser Studie sollte die Effektivität und Sicherheit einer neuen Generation ballonexpandierbarer Stents getestet werden. Dazu wurden Patienten mit einer symptomatischen intrakraniellen Stenose $(70-99 \%)$ innerhalb der letzten
30 Tage eingeschlossen. Alle Teilnehmer erhielten eine medikamentöse Therapie bestehend aus Clopidogrel (75 mg/Tag) für die ersten drei Monate sowie Acetylsalicylsäure (81-325 mg/Tag) lebenslänglich. Zusätzlich wurden alle individuellen kardiovaskulären Risikofaktoren leitliniengerecht behandelt.

In der Interventionsgruppe wurde zusätzlich ein intrakranieller ballonexpandierbarer Stent gelegt. Primärer Outcome waren erneuter Hirninfarkt oder TIA im betroffenen Infarktareal innerhalb eines Jahres. Als Sicherheitsendpunkte wurden Hirninfarkt in einem anderen Infarkt- 